\title{
Roots of quantum computing supremacy: superposition, entanglement, or complementarity?
}

\author{
Andrei Khrennikov ${ }^{\mathrm{a}}$ \\ International Center for Mathematical Modeling in Physics and Cognitive Sciences, Linnaeus University, 35195 Växjö, \\ Sweden
}

Received 12 March 2020 / Accepted 5 January 2021 / Published online 13 April 2021

(C) The Author(s) 2021

\begin{abstract}
The recent claim of Google to have brought forth a breakthrough in quantum computing represents a major impetus to further analyze the foundations for any claims of superiority regarding quantum algorithms. This note attempts to present a conceptual step in this direction. I start with a critical analysis of what is commonly referred to as entanglement and quantum nonlocality and whether or not these concepts may be the basis of quantum superiority. Bell-type experiments are then interpreted as statistical tests of Bohr's principle of complementarity (PCOM), which is, thus, given a foothold within the area of quantum informatics and computation. PCOM implies (by its connection to probability) that probabilistic algorithms may proceed without the knowledge of joint probability distributions (jpds). The computation of jpds is exponentially time consuming. Consequently, classical probabilistic algorithms, involving the computation of jpds for $n$ random variables, can be outperformed by quantum algorithms (for large values of $n$ ). Quantum probability theory (QPT) modifies the classical formula for the total probability (FTP). Inference based on the quantum version of FTP leads to a constructive interference that increases the probability of some events and reduces that of others. The physical realization of this probabilistic advantage is based on the discreteness of quantum phenomena (as opposed to the continuity of classical phenomena).
\end{abstract}

\section{Introduction}

The recent tremendous success in engineering of quantum computers generated the new wave of interest to foundational analysis of their functioning. This interest is heated by widely distributed in media claims that they beat classical digital computers as well as intensive critique of such claims - especially, the recent Googleteam claim $[1,2]$ (and its questioning in $[3,4]$ ). This type of controversy cannot be resolved just my making large investments and hype in the new media. Unfortunately, the foundational grounds of quantum computing are really shaky. ${ }^{1}$

The possibility to process superpositions is often pointed as basic for quantum computing. However, classical wave devices can also generate superpositions, e.g., optical computers. So, superposition (treated straightforwardly) cannot lead to computational supremacy (see Sect. 3, classical vs. quantum superpositions).

Typically entanglement is considered as crucial for quantum computing. However, entanglement is just a mathematical structure and its physical meaning is still

1 We mean really foundational grounds, not formal mathematical propositions.

\footnotetext{
a e-mail: Andrei.Khrennikov@lnu.se (corresponding author)
}

the subject of intensive foundational debates related to such an ambiguous notion as quantum nonlocality. We can point to two main tendencies in treatment of entanglement: (1) using the formal mathematical definitionstate's non-separability; and (2) sanctifying entanglement (and quantum computing) by referring to quantum nonlocality.

To avoid involvement into deep foundational discussions, typically researchers working on quantum computing theory use the first strategy and the essential part of this theory is simply linear algebra in complex Hilbert space. One of our aims is to show that the formal mathematical treatment of entanglement cannot justify supremacy of quantum computing. Following recent paper [5], we criticize common referring to quantum nonlocality as the physical basis of entanglement. We elevate the role of the Bohr's principle of complementarity (PCOM) [7] by interpreting the Bell experiments as statistical tests of this principle (Sect. 3).

This principle is endowed with the information interpretation (see also Plotnitsky and Jaeger [8-12]). The latter has various versions, some of them are coupled to traditional interpretations of QM, as say the Copenhagen and statistical ones; others were elaborated within quantum information theory such as Växjö interpretation (VXI) [13], Quantum Bayesianism (QBism)_Fuchs et al. [14], and RTW (Reality without 
Realism)—Plotnitsky, see, e.g., [15] for detailed presentation and foundational discussion. VXI combines the statistical interpretation with contextuality: QM describes probability update for context transition. ${ }^{2} \mathrm{In}$ this paper, we do not stress the update component of VXI; the reader can proceed with the statistical interpretation: A quantum state represents symbolically an ensemble of identically prepared systems. At the same time, we shall use the terminology "the state of a system", but with above statistical meaning.

Bohr did not use with the statistical interpretation and the "Copenhagen interpretation" is typically understood as the individual interpretation, i.e., a quantum state is the state of the concrete system. However, the basis of Bohr's formulation of PCOM is interpretation-invariant (see PCOM 1-PCOM3, Sect. 4). Bohr's views can be easily adapted to the statistical interpretation. The latter matches best with QPT and its applications to quantum computing.

The main distinguishing feature of QPT is processing of probabilistic data without computing the joint probability distribution (jpd). Jpd's calculation is exponentially time consuming. Consequently, classical probabilistic algorithms involving calculation of jpd for $n$ random variables can be over-performed by quantum algorithms (for big values of $n$ ). QPT modifies the classical formula of total probability (FTP). Inference based on the quantum version of FTP leads to constructive interference increasing probabilities of some events. In QPT, this interference of probabilities is obtained via operation with superpositions. However, as was already mentioned, superposition by itself is also a feature of classical waves and it cannot lead to computational supremacy. The basic feature of quantum computing is the possibility to extract discrete observations from a quantum state, in Bohr's terminology, quantum phenomena. This discreteness is a consequence of the existence individual quantum systems, say photons (in contrast to continuous beams of classical light).

\section{Superposition: classical vs. quantum}

Quantum and classical superpositions were compared by Dirac [16], p. 13: "The nonclassical nature of the superposition process is brought out clearly if we consider the superposition of two states $A$ and $B$, such that there exists an observation which, when made on the system instate $A$, is certain to lead to one particular result, a say, and when made on the system in state $B$, is certain to lead to some different result, $b$ say. What will be the result of the observation when made on the system in the superposition state? The answer is that the result will be sometimes a and sometimes $b$, depending on the relative weights of $A$ and B.". Dirac clearly pointed to the crucial role of observation in the interpretation of quantum superposition (see also Jaeger [10], p. 1) and its characteristic feature: its ability to produce "one particular result." This discreteness of quantum

2 QBism also emphasizes update, but within subjective probability theory. observations was formalized by Bohr as the notion of phenomenon $[7,9,11]$ (Sect. 4). The possibility to extract the individual value from a signal plays the crucial role in quantum computing. Thus, superposition is basic for quantum computing, but it has to be understand in the right way as the genuine quantum superposition.

\section{Entanglement and quantum nonlocality or complementarity of quantum observables?}

We turn to aforementioned two behavioral tendencies in handling of entanglement, see introduction. The formal mathematical treatment of entanglement is convenient (especially in quantum engineering), but it leads to misunderstanding of the real physical situation. We illustrate this problem by referring to studies on classical entanglement (see review [17]). The mathematical structure for a few degrees of freedom of the classical electromagnetic field is identical to its counterpart for a compound genuine quantum system. Entangled (in the mathematical sense) states can be generated by beams of classical light. This means that the strategy based on the formal mathematical treatment of entanglement (without its physical interpretation) was not successful. And commonly, the second behavioral tendency is in use: quantum systems are so unusual that they can have really mystical features, e.g., such as a spooky action at a distance- quantum nonlocality.

However, the notion of quantum nonlocality is ambiguous (see, e.g., [18]). This notion is a mixture of quantum and subquantum considerations culminating in the Bell-type inequalities. In discussions, nonlocality of genuine quantum theory is mixed with possible nonlocality of hypothetical subquantum models. The first one is combination of the Lüders projection postulate and the individual interpretation of a quantum state. The second one is formulated in terms of hidden variables and operationally represented as violation the Bell-type inequalities. The first step to clarification should be separation of two types of nonlocality [5] (see also [6] for detailed classification). The projection nonlocality can be eliminated from quantum theory by endowing it with the statistical interpretation. What is about violation of the Bell inequalities? Although consideration of hidden variables may be interesting for foundations, in real applications, say to quantum computing, it is natural to use solely the standard quantum theory and, in particular, to treat the Bell-type inequalities in the purely quantum mechanical framework. In recent article [5], I demonstrated that local incompatibility of observables, i.e., observables $A_{j}, j=1,2$, of Alice (and $B_{j}, j=1,2$, of Bob) is necessary and sufficient for violation of the $\mathrm{CHSH}$-inequality for at least one quantum state. Mathematically local incompatibility is described as noncommutativity of the operators representing observables: $\left[A_{1}, A_{2}\right] \neq 0$ and $\left[B_{1}, B_{2}\right] \neq 0$. Thus, Bell's type inequalities can be interpreted as the special statistical tests of PCOM [5]. Quantum mechanics is a local theory constrained by PCOM: there is no need neither in spooky action at a distance nor in consideration of nonlocal theories with hidden variables to explain violation of Bell inequality. In the light of the critical argumentation [5], the quantum nonlocality interpretation of entanglement lost 
its power. The main operational consequence of this argumentation is that entanglement should be treated not as "entanglement of systems", but as entanglement of (incompatible) observables.

\section{Complementarity principle}

In 1949, Bohr ([7], v. 2, p. 40-41) presented the essence of complementarity in the following widely cited statements: "This crucial point ... implies the impossibility of any sharp separation between the behavior of atomic objects and the interaction with the measuring instruments which serve to define the conditions under which the phenomena appear. In fact, the individuality of the typical quantum effects finds its proper expression in the circumstance that any attempt of subdividing the phenomena will demand a change in the experimental arrangement introducing new possibilities of interaction between objects and measuring instruments which in principle cannot be controlled. Consequently, evidence obtained under different experimental conditions cannot be comprehended within a single picture, but must be regarded as complementary in the sense that only the totality of the phenomena exhausts the possible information about the objects."

"While the combination of these concepts [space-time representations and dynamical conservation laws] into a single picture of a causal chain of events is the essence of classical mechanics, room for the regularities beyond the grasp of such a description is just afforded by the circumstance that the study of the complementarity phenomena demands mutually exclusive experimental arrangements."

By PCOM, observables do not deliver the genuine properties of quantum systems. Their outputs result from the interaction of a system $S$ and a measurement instrument $M$. PCOM implies impossibility to separate features of a system $S$ from interaction with a measurement instrument $M$. This implies the existence of complementary complexes of experimental conditions (contexts). Logically, there is no reason to assume that information obtained for different contexts can be always consistently comprehended within a single theoretical picture. Complementarity is often treated as physical impossibility of joint measurements, say of position and momentum. However, from the citation it is clear that, although Bohr did not write about information, his statement is informational per its nature (Sect. 5).

PCOM has another part that is also encoded in Bohr's statement, as "the individuality of the typical quantum effects". This is discreteness of outputs of quantum observables: individuality of the act of measurement, say the click of a photo-detector with the possibility to couple this click with the individual photon. This individual act is called phenomenon (see Bohr $[7,9,11]$ ). Discreteness of detection events is the fundamental feature of quantum physics justifying existence of quantum systems, carriers of quanta [18]. This is the main distinguishing feature of genuine quantum superposition - superposition of concrete outputs of observations.

Thus, PCOM can be presented as following five interconnected statements (see also [19]):

- PCOM 1 Inseparability: Dependence of measurement's output on the experimental context.

- PCOM 2 Complementarity: Existence of complementary experimental contexts.
- PCOM 3 Individuality: Discreteness of quantum measurements.

Application of these principles is constrained by the interpretation of a quantum state; we use the statistical interpretation (in fact, VXI).

Now, we discuss the last part of Bohr's formulation of PCOM, "... the totality of the phenomena exhausts the possible information about the objects." Using the Copenhagen interpretation, Bohr coupled this statement to individual objects. However, we want to use the statistical interpretation. Then, Bohr's statement can be understood is as follows: using complementary experimental contexts, we are able to collect the complete information about a quantum state, as representing an ensemble of identically prepared quantum systems. And we can complete the above list by the following principle:

- PCOM 4 Observational Completeness: Complementary observations provide complete information about a quantum state.

\section{Quantum supremacy from QPT}

We now analyze PCOM 2 in more detail. We proceed with its information interpretation that is the most appropriate for quantum computing (see [21] and especially [8]). As we know, information is firmly coupled to probability. Hence, PCOM 2 is about the impossibility of construction of the comprehensive probabilistic description of data collected through measurements of a few arbitrary quantum observables. Observables for which such description is possible are called compatible and those for which it is impossible are called incompatible. However, the meaning of "comprehensive probabilistic description" cannot be specified without specification of a mathematical model of probability. And it seems to be natural to follow Kolmogorov [22] (1933) and consider his measure - theoretical model of probability.

The Kolmogorov probability space [22] is any triple $(\Lambda, \mathcal{F}, P)$, where $\Lambda$ is a set of random parameters, $\mathcal{F}$ is a $\sigma$-algebra of its subsets, and $P$ is a probability measure on $\mathcal{F}$. Probability measure is countably additive. Another basic feature is the introduction of conditioning by the Bayes' formula $p(B \mid A) \equiv p(B \wedge A) / P(A), P(A)>0$. Observables are represented by random variables. For any group of random variables $A_{1}, \ldots, A_{n}: \Lambda \rightarrow \mathbf{R}$, their joint probability distribution (jpd) is well defined, $p_{A_{1} \ldots A_{n}}\left(x_{1}, \ldots, x_{n}\right)=P(\lambda \in \Lambda$ : $\left.A_{1}(\lambda)=x_{1}, \ldots, A_{n}(\lambda)=x_{n}\right)$. Moreover, to operate consistently, we have to compute jpds for all possible combinations of observables. They can be found as marginals of $p_{A_{1} \ldots A_{n}}$, The classical probability version of PCOM 4 is that probability measure $P$ represents the complete state of a system.

Now, we briefly recall the basics of quantum calculus of probability (see, e.g., $[13,23,24]$ ), to compare with classical probability. Consider a pure quantum state $\psi$ and quantum observables $A$ and $B$ with discrete spectra. For simplicity, we consider representation of observables by Hermitian operators with nondegenerate spectra; $\left(\left|\alpha_{i}\right\rangle\right)$ and $\left(\left|\beta_{i}\right\rangle\right)$ denote their eigenbases. Quantum probability is defined by Born's rule: $q\left(B=\beta_{i}\right) \equiv q\left(B=\beta_{i} ; \psi\right)=\left|\left\langle\psi \mid \beta_{i}\right\rangle\right|^{2}, q\left(A=\alpha_{i}\right) \equiv$ $q\left(A=\alpha_{i} ; \psi\right)=\left|\left\langle\psi \mid \alpha_{i}\right\rangle\right|^{2}$. Quantum conditional probability $q\left(B=\beta_{i} \mid A=\alpha_{j}\right)$ is defined as the probability w.r.t. the post-measurement state for output $A=\alpha_{j}$. In the case of 
non-degenerate spectrum of $A$, this state is simply eigenvector $\left|\alpha_{j}\right\rangle$ and $q\left(B=\beta_{i} \mid A=\alpha_{j}\right)=\left|\left\langle\beta_{i} \mid \alpha_{j}\right\rangle\right|^{2}$.

The impossibility to define jpds (see PCOM 2) for incompatible quantum observables led to discovery of the novel probability calculus, quantum probability theory. Here, instead of manipulating with jpds, one uses quantum state vectors and their transformations. The algorithmic power of quantum probability is also coupled to PCOM 4. By operating with incompatible observables, one can extract complete information about the state of a quantum system. (We recall that in classical probability calculus complete information is encoded in jpd.) Moreover, by PCOM 3 this information can be represented in the form of discrete events, phenomena. The latter is important for the frequency realization of probability playing the crucial role in quantum computing.

Of course, the quantum probability theory was not just a mathematical discovery of a nonclassical probability model (similar to the discovery by Lobachevsky of non-Euclidean geometry). The crucial point is that creation of the quantum probability theory was based on the discovery of a wide class of physical systems following the laws of this theory. The most important feature of these systems is represented by PCOM 4. Complete information about the state of a quantum system can be extracted from separate measurements of quantum observables. In contrast to classical systems, for quantum systems, there is no need in joint measurements.

In the classical model of probability, all observables can be represented as random variables with respect to the same probability measure $P$. Additivity and Bayes' conditioning imply the formula of total probability (FTP). Consider two discrete random variables $A=\alpha_{1}, . ., \alpha_{n}$ and $B=\beta_{1}, \ldots, \beta_{n}$. Then $p\left(B=\beta_{i}\right)=\sum_{j} p\left(A=\alpha_{j}\right) p\left(B=\beta_{i} \mid A=\alpha_{j}\right)$. In QPT, FTP is violated (see, e.g., $[13,24]$ ). It is transformed into FTP with the interference term (a perturbation of the classical formula). By expanding $\psi$ with respect to orthonormal bases consisting of eigenvectors of $A$ and $B$, we obtain $[13,24]$ :

$$
\begin{aligned}
& q\left(B=\beta_{i} ; \psi\right)=\sum_{j} q\left(A=\alpha_{j} ; \psi\right) q\left(B=\beta_{i} \mid A=\alpha_{j}\right) \\
& \quad+2 \sum_{k<j} \cos \theta_{k j} \sqrt{q\left(A=\alpha_{j}\right) q\left(B=\beta_{i} \mid A=\alpha_{j}\right)},
\end{aligned}
$$

where the additional parameter $\theta_{k j}$ are combination of phases of $\psi$ and eigenvectors of observables $A, B$.

Transformation of classical FTP into quantum FTP is one of the main roots of quantum superiority. If the interference term is positive (constructive interference of probabilities), then

$$
q\left(B=\beta_{i}\right)-p\left(B=\beta_{i}\right)>0,
$$

(the quantum and classical probabilities are given by corresponding FTPs). Thus, an algorithm exploring QPT can essentially increase the probability of some event. We stress that this can happen only by exploring incompatible quantum observables. They are represented by noncommuting operators.

The computational power of operating with incompatible observables is based on PCOM 4: the possibility to extract the complete information about system's state with the aid of such observables. So, any event $E$ can be represented in the form $E=\left\{B=\beta_{i}\right\}$ for some quantum observable $B$.

\section{Quantum versus classical inference}

Bayes' formula for conditioning and FTP are the basic elements of classical probability update, the procedure known as inference. QPT represents a different procedure for probability inference. This new form of probability update leads to assigning to event $\left\{B=\beta_{i}\right\}$ probability $q\left(B=\beta_{i}\right)$ that can be higher than probability $p\left(B=\beta_{i}\right)$ obtained via classical probability update.

Quantum FTP (1) can lead to increase of probability for some event. However, this is just the formal explanation of quantum superiority. Yes, mathematics implies (2). What does it mean from the computational viewpoint? Here we point that the basic feature of QPT is the possibility to proceed without calculation of jpds. This possibility to get rid of calculations of jpds in combination with quantum conditioning via state's projection is the origin of probability increase, see (2), implied by the quantum probability calculus. This is the computational basis of supremacy of quantum algorithms.

Our previous considerations lead to the following hypothesis: The essence of "quantum superiority" in the use of nonclassical probability inference. In connection with this hypothesis, the natural question arises: Can one approach superiority over some classical probabilistic algorithms using nonclassical probability inferences different from the quantum inference? Formally, it seems to be possible and this is an interesting topic for studies in computer science. This is the good place to remark that, although simple derivation of FTP with the interference term is based on Hilbert space, this formula can be derived without any coupling to linear space representation. Such quantum-like FTP can be derived (see $[13,24]$ ) on the basis of contextual probability theory. which provides the general probabilistic representation of PCOM.

\section{Concluding remarks}

In the light of controversy generated by Google's claim on approaching superiority of quantum computer over classical digital computer, it is important to reanalyze the foundational grounds of the possibility of such superiority. Typically, the latter is associated with entanglement (quantum nonlocality, spooky action at a distance) or straightforwardly interpreted superposition. However, after the recent article [5] representing the PCOM-based interpretation of violation of the Bell type inequalities and demonstrating locality of quantum mechanics, the mystical nonlocality argument lost its power. The crucial difference between the classical (wave type) and genuine quantum superpositions was highlighted in [18].

In this note, we coupled quantum computational supremacy with quantum complementarity, PCOM. The information interpretation of PCOM 2 (existence of complementary experimental contexts) and PCOM 4 (complementary observations provide complete information about system's state) lead to their probabilistic reinterpretation.

By analyzing the difference between classical and quantum probability calculi, we point to calculation of jpds (joint probability distributions) as the main source of consuming of computational resources in classical probabilistic algo- 
rithms. The quantum calculus can function without calculation of jpds, without this (exponential) time consuming. This calculus opens the door to a new way of probability update, via quantum state update. This non-Bayesian inference is based on the modification of FTP expressing interference of probabilities. Constructive interference can essentially increase probability of some events, comparing with classical Bayesian inference. This constructive interference is the main root of quantum computational superiority.

We also elevate the role of the frequency realization of probability (mathematically the law of large numbers) and its coupling with PCOM 3, the basic distinguishing feature of quantum measurements - extraction of discrete phenomena.

We can conclude that in principle algorithms based on QPT can demonstrate superiority over corresponding classical probabilistic algorithms, via saving computational resources by eliminating jpds-calculations and by exploring quantum inference, based on the constructive interference of probabilities.

The role of linear space representation and linear dynamics of measurement in realization of supremacy of quantum computations was analyzed in preprint [20]. However, as was emphasized in this preprint, the complete analysis would be possible only after solution of the measurement problem [25] (see $[26,27]$ for the recent progress).

Funding Information Open access funding provided by Linnaeus University.

Open Access This article is licensed under a Creative Commons Attribution 4.0 International License, which permits use, sharing, adaptation, distribution and reproduction in any medium or format, as long as you give appropriate credit to the original author(s) and the source, provide a link to the Creative Commons licence, and indicate if changes were made. The images or other third party material in this article are included in the article's Creative Commons licence, unless indicated otherwise in a credit line to the material. If material is not included in the article's Creative Commons licence and your intended use is not permitted by statutory regulation or exceeds the permitted use, you will need to obtain permission directly from the copyright holder. To view a copy of this licence, visit http://creativecomm ons.org/licenses/by/4.0/.

\section{References}

1. F. Arute et al., Nature 574, 505-510 (2019)

2. J. Martinis, S. Boixo, Quantum supremacy using a programmable superconducting processor, (2019), posted on Wednesday, October 23, 2019, accessed on October 28th, (2019)
3. E. Pednault, J.A. Gunnels, G. Nannicini, L. Horesh, R. Wisnieff, (n.d.) arXiv:1910.09534

4. K. Svozil, (n.d.) arXiv:1911.00577

5. A. Khrennikov, Get rid of nonlocality from quantum physics. Entropy 21(8), 806 (2019)

6. K. Hess, J. Modern Phys. 10, 1209-1221 (2019)

7. N. Bohr, The Philosophical Writings of Niels Bohr (Ox Bow Press, Woodbridge, 1987)

8. A. Plotnitsky, Quantum Atomicity and Quantum Information: Bohr, Heisenberg, and Quantum Mechanics as an Information Theory, in Quantum Theory: Reconsideration of Foundations (Växjö Univ. Press, Växjö, 2002), pp. 309-343

9. A. Plotnitsky, Reading Bohr: Physics and Philosophy (Springer, Heidelberg, 2006)

10. G. Jaeger, Quantum Information. An Overview (Springer, Heidelberg, 2007)

11. A. Plotnitsky, Niels Bohr and Complementarity: An Introduction (Springer, Berlin, 2012)

12. G. Jaeger, Quantum Objects: Non-Local Correlation, Causality and Objective Indefiniteness in the Quantum World (Springer, Berlin-New York, 2013)

13. A. Khrennikov, Probability and Randomness: Quantum Versus Classical (Imperial College Press, London, 2016)

14. C.A. Fuchs, N.D. Mermin, R. Schack, Am. J. Phys. 82, 749-759 (2014)

15. A. Plotnitsky, A. Khrennikov, Found. Phys. 45, 1269 $1300(2015)$

16. P. Dirac, The Principles of Quantum Mechanics, 4th edn. (Clarendon Press, Oxford, 2012)

17. N. Korolkova, G. Leuchs, Rep. Progr. Phys. 82, 056001 (2019)

18. A. Khrennikov, Found. Phys., https://link.springer. com/article/10.1007/s10701-020-00319-7

19. A. Khrennikov, Open Phys. 15, 734-738 (2017)

20. A. Khrennikov, (2019). arXiv:1911.10337 [quant-ph]

21. A. Khrennikov (ed.), Quantum Theory: Reconsideration of Foundations (Växjö Univ. Press, Växjö, 2002)

22. A.N. Kolmolgoroff, Grundbegriffe der Wahrscheinlichkeitsrechnung (Springer, Berlin, 1933)

23. M. Ozawa, New Gen. Comput. 34, 125-152 (2016)

24. A. Khrennikov, Contextual Approach to Quantum Formalism (Springer, Berlin-New York, 2009)

25. J. von Neuman, Mathematical Foundations of Quantum Mechanics (Princeton Univ. Press, Princenton, 1955)

26. A. Allahverdyan, R. Ballian, Th. M. Nieuwenhuizen 376, 324-352 (2017)

27. A. Allahverdyan, R. Ballian, T.M. Nieuwenhuizen, Phys. Rep. 525, 1-166 (2013) 